\title{
Error-Induced Blindness: Error Detection Leads to Impaired Sensory Processing and Lower Accuracy at Short Response-Stimulus Intervals
}

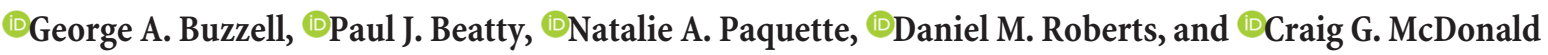 \\ Psychology Department, George Mason University, Fairfax, Virginia 22030
}

\begin{abstract}
Empirical evidence indicates that detecting one's own mistakes can serve as a signal to improve task performance. However, little work has focused on how task constraints, such as the response-stimulus interval (RSI), influence post-error adjustments. In the present study, event-related potential (ERP) and behavioral measures were used to investigate the time course of error-related processing while humans performed a difficult visual discrimination task. We found that error commission resulted in a marked reduction in both task performance and sensory processing on the following trial when RSIs were short, but that such impairments were not detectable at longer RSIs. Critically, diminished sensory processing at short RSIs, indexed by the stimulus-evoked P1 component, was predicted by an ERP measure of error processing, the Pe component. A control analysis ruled out a general lapse in attention or mind wandering as being predictive of subsequent reductions in sensory processing; instead, the data suggest that error detection causes an attentional bottleneck, which can diminish sensory processing on subsequent trials that occur in short succession. The findings demonstrate that the neural system dedicated to monitoring and improving behavior can, paradoxically, at times be the source of performance failures.
\end{abstract}

Key words: attention; ERN; error; Pe; post-error accuracy; post-error slowing

\section{Significance Statement}

The performance-monitoring system is a network of brain regions dedicated to monitoring behavior to adjust task performance when necessary. Previous research has demonstrated that activation of the performance monitoring system following incorrect decisions serves to improve future task performance. However, the present study provides evidence that, when perceptual decisions must be made rapidly (within approximately half a second of each other), activation of the performance-monitoring system is predictive of impaired task-related attention on the subsequent trial. The data illustrate that the cognitive demands imposed by error processing can interfere with, rather than enhance, task-related attention when subsequent decisions need to be made quickly.

\section{Introduction}

Previous investigations of the human performance-monitoring system (Botvinick et al., 2001; Holroyd and Coles, 2002) suggest that the detection of mistakes serves as a signal to adapt and improve ongoing behavior. However, behavioral work has challenged the notion that error detection universally leads to enhanced cognitive control (Jentzsch and Dudschig, 2009; Notebaert et al., 2009). One possible explanation for this discrepancy is that the error detection reflects a resource-intensive process, which can interfere with task

\footnotetext{
Received April 10, 2016; revised Jan. 30, 2017; accepted Feb. 4, 2017.

Author contributions: G.A.B., D.M.R., and C.G.M. designed research; G.A.B.,P.J.B., and N.A.P. performed research; G.A.B., P.J.B., and C.G.M. analyzed data; G.A.B., P.J.B., N.A.P., D.M.R., and C.G.M. wrote the paper.

This work was supported by the Air Force Office of Scientific Research Grant FA9550-10-1-0385 and the Center of Excellence in Neuroergonomics, Technology, and Cognition.

We declare no competing financial interests.

Correspondence should be addressed to Dr. George A. Buzzell, George Mason University MS 3F5, 4400 University Drive, Fairfax, VA 22003. E-mail: gbuzzell@gmu.edu.

DOI:10.1523/JNEUROSCI.1202-16.2017

Copyright $\odot 2017$ the authors $\quad 0270-6474 / 17 / 372895-09 \$ 15.00 / 0$
}

performance in situations where decisions must be made rapidly. The current report used the event-related potential (ERP) technique and manipulated the time between error commission and subsequent stimulus presentation (response-stimulus interval [RSI]) to investigate the possible time course of attentional distraction caused by error detection.

Error-commission is associated with activation of the cingulate and medial-frontal cortex (MFC) (Ridderinkhof et al., 2004), observable in ERP recordings as the fronto-central error-related negativity (ERN), followed by a centro-parietal error positivity (Pe) (Ullsperger et al., 2014). Error-related activity within MFC has been linked to post-error slowing (PES) (Botvinick et al., 2001), post-error accuracy (PEA) increases (Maier et al., 2011), and enhancement of task-relevant sensory processing (King et al., 2010). However, all of these previous investigations used RSIs $>1000 \mathrm{~ms}$. Therefore, existing support for the notion that errors lead to increased control has left open the question of whether error-induced control is limited to situations in which RSIs are relatively long. 
A key challenge to the notion that error detection universally leads to enhanced control has come from a behavioral study by Jentzsch and Dudschig (2009), which directly investigated the influence of RSI on post-error behavior. At RSIs $\sim<500 \mathrm{~ms}$, errors led to a reduction in PEA, whereas at RSIs of $1000 \mathrm{~ms}$, PEA did not significantly differ. These data are suggestive of error detection causing an attentional bottleneck, which impairs subsequent task performance when the RSI is short. However, neural evidence linking error processing to impaired attentional processing is lacking.

Theoretical models of executive control suggest that adequate performance in difficult perceptual decision making tasks requires enhanced attentional control over sensory cortex (Lavie, 1995; de Fockert et al., 2001). One of the earliest ERP components consistently influenced by spatial attention (Hillyard et al., 1998) and featurebased attention for color (Zhang and Luck, 2009) is the lateraloccipital P1 component. If error detection does produce an attentional bottleneck, one might expect a reduction of $\mathrm{P} 1$ on posterror trials. However, impaired sensory attention following errors could also be explained by a general lapse in attention causing both error commission and modulation of post-error processing. If error detection itself can be the source of an attentional bottleneck, then $\mathrm{P} 1$ reductions on post-error trials should be selectively predicted by the extent of error-related processing. In contrast to the ERN, the Pe appears to be a good candidate for driving such an attentional bottleneck, given that this component has been linked to the resourceintensive process of consciously deciding whether an error has occurred (Nieuwenhuis et al., 2001; Steinhauser and Yeung, 2010).

In the present investigation, participants performed a difficult color-discrimination task in which post-error behavior and electrophysiological responses were compared at relatively short (200-533 ms) and long (866-1200 ms) RSIs. In line with recent investigations (Jentzsch and Dudschig, 2009), we predicted that PEA decreases would be maximal at short RSIs. Critically, we also expected the stimulus-locked P1 following errors to be diminished at short RSIs, suggesting impairment in sensory attention, but that impairments would be alleviated at longer RSIs. In accordance with the notion that errors directly cause an attentional bottleneck, we hypothesized that the magnitude of error-related processing, as indexed by the Pe component of the ERP, would be negatively related to sensory processing on the subsequent trial.

\section{Materials and Methods \\ Participants}

Twenty-five students from George Mason University participated in the study in exchange for course credit. The sample size for this study was determined by conducting power analyses, which leveraged previous investigation of the P1 (Zhang and Luck, 2009) and Pe (Steinhauser and Yeung, 2010) ERP components to estimate effect sizes. Based on power analyses of the P1 effect, it was determined that 19 participants would yield $80 \%$ power for detecting a P1 effect, with considerably fewer being required to provide comparable power for the Pe; data from 25 participants were collected to ensure that at least 20 participants remained for statistical analyses following potential rejection of participants for poor behavioral performance and/or excessive EEG artifacts. One participant was removed due to noisy EEG; one participant was removed due to low accuracy (accuracy $<60 \%, 2$ SD below the mean). A total of 23 participants ( 8 male, mean $\pm S D$ age, $22.0 \pm 5.0$ years) remained for behavioral and electrophysiological analyses. All participants were right-handed, tested to ensure normal (or corrected-to-normal) visual acuity and color vision, had no known neurological deficits, and were not currently taking any medications known to affect the CNS. All participants provided written informed consent before participation, and all procedures were approved by the George Mason University Office of Research Integrity and Assurance.

\section{Procedure and task design}

Participants performed a difficult two-choice perceptual decisionmaking task, in which the color of two simultaneously presented concentric circles had to be discriminated (Fig. 1). On each trial, two concentric circles were presented for $200 \mathrm{~ms}$, and participants were required to respond with the index finger of one hand if the circles were the same color, and the index finger of the other hand if the circles differed in color; the concentric circles were the same color on $50 \%$ of trials. The response mappings between stimuli and hand were counterbalanced across individuals. Participants were instructed to respond as quickly and accurately as possible before a $2000 \mathrm{~ms}$ response deadline. If participants responded after the $2000 \mathrm{~ms}$ response deadline, a "too slow" message appeared on the screen and this trial (and the subsequent trial) was removed from further analyses. Additional feedback of response accuracy was provided during practice sessions; however, no other trial-bytrial feedback was provided during the experimental task. Following response, a randomly selected RSI preceded the presentation of the subsequent trial; RSIs were drawn from a uniform distribution ranging from 200 to $1200 \mathrm{~ms}$. These RSI values are based on previous research investigating the influence of RSI on PES and PEA (Jentzsch and Dudschig, 2009). Given that some trials were separated by an RSI interval as short as $200 \mathrm{~ms}$, an a priori decision was made to not allow participants to correct their responses in the present task. That is, participants were explicitly instructed not to correct their response, even if they knew they made a mistake. Participants completed 20 experimental blocks consisting of 84 trials each (1680 trials total). To minimize fatigue, a mandatory $30 \mathrm{~s}$ break was required after each block. Additionally, participants performed a passive version of the task every six blocks, in which only a simple response was made to stimulus presentation and no discrimination was required; analysis of this passive task is not discussed here.

Before completing the experimental task, participants were familiarized with the task by practicing 20 trials of the task in which the color difference between the two concentric circles was easy to distinguish, with accuracy feedback after every trial. Participants then completed 80 trials of an adaptive version of the task (without feedback) to titrate task difficulty (difference in hue between the two concentric circles) to $\sim 80 \%$. Following the titration procedure, participants practiced 40 trials of the experimental task (without feedback); if performance was not within $\pm 5 \%$ of the desired accuracy rate, then the calibration procedure was performed a second time to ensure $\sim 80 \%$ accuracy for all partic- 
ipants. Participants were then fitted with an EEG cap while they completed a series of questionnaires. Following EEG preparation, resting-state EEG (eyes-open and eyes-closed) was recorded and an additional practice task ( 40 trials, without feedback) was completed before the beginning the experimental task. Following task completion, an additional series of questionnaires was completed and participants were debriefed.

\section{Stimuli}

Stimuli consisted of a green (8-bit sRGB: $r=0.118, \mathrm{~g}=0.392, \mathrm{~b}=0$; $\mathrm{cd} / \mathrm{m}^{2}=54.846$ ) circle (subtending 2 degrees) with a smaller circle (subtending 1.12 degrees) superimposed within the center of the larger circle. The smaller circle could either be the same color as the larger circle or differ slightly in hue as to appear slightly yellowish-green (mean 8-bit sRGB: $\left.r=0.144 \mathrm{~g}=0.384, \mathrm{~b}=0 ; \mathrm{cd} / \mathrm{m}^{2}=54.854\right)$. Stimuli were presented on a gray background $\left(\mathrm{cd} / \mathrm{m}^{2}=38.72\right)$ within the center of a light gray box $\left(\mathrm{cd} / \mathrm{m}^{2}=70.41\right)$, subtending $3.75 \times 3.75$ degrees, which remained on screen throughout each experimental block. The difference in hue between the "same" and "different" stimuli was titrated for each participant to achieve $\sim 80 \%$ accuracy using the QUEST psychophysical threshold procedure (Brainard, 1997; Pelli, 1997; Kleiner et al., 2007). The "same" and "different" stimuli were closely matched for luminance; across participants, the average difference in luminance was $0.008 \mathrm{~cd} / \mathrm{m}^{2}$. Stimuli were generated using the MATLAB programming environment (The MathWorks), Psychtoolbox functions (Brainard, 1997; Pelli, 1997; Kleiner et al., 2007), and custom MATLAB scripts. Stimuli were presented on a standard LCD desktop monitor.

A control analysis of the "same" and "different" stimuli was conducted to confirm no significant differences in terms of the elicited P1 response; all experimental trials, regardless of behavioral response, were sorted as a function of stimulus type, and the stimulus-locked P1 component was statistically compared. Mirroring the primary analyses presented in the section entitled "ERP analyses," P1 was defined as mean amplitude during a 124-164 ms time window, collapsed across electrodes PO7 and PO8. A repeated-measures $t$ test revealed no significant difference in P1 amplitude between the "same" and "different" stimuli $\left(t_{(1,22)}=0.0004\right.$, $p=0.997)$.

\section{EEG recording procedures}

EEG data were collected using a Neuroscan SynAmps2 amplifier and SCAN 4.01 acquisition software (Compumedics). Data were collected from 64 in-cap $\mathrm{Ag} / \mathrm{AgCl}$ electrodes, following the extended 10-20 arrangement, and two additional electrodes placed over the left and right mastoids. In addition, electro-oculogram was collected from two pairs of bipolar electrode montages located above and below the left eye, as well as over the outer canthus of each eye. During data acquisition, the 64 in-cap electrodes, as well as the two mastoid electrodes, were referenced to an in-cap reference located between electrodes $\mathrm{Cz}$ and $\mathrm{CPz}$. All electrodes were recorded using an in-cap ground located just anterior to Fz. EEG data were collected at a sampling rate of $1000 \mathrm{~Hz}$ and using an online bandpass filter of $0.1-250 \mathrm{~Hz}$. Impedance for all electrodes was maintained $<5 \mathrm{k} \Omega$ throughout the recording session.

\section{Behavioral analyses}

For all analyses, trials in which participants did not respond, responded before $150 \mathrm{~ms}$, or responded after the $2000 \mathrm{~ms}$ response deadline, were removed from the analyses. Overall accuracy, as well as correct and error response times (RTs) were calculated; RTs for correct and error responses were statistically compared using a repeated-measures $t$ test. PES was defined as the percentage change in RT for correct trials following errors, relative to each individual's baseline RT for correct trials following correct responses. Similarly, PEA was defined as the percentage change in accuracy for responses following errors, relative to each individual's baseline accuracy for responses following correct trials. PES and PEA were separately calculated for short (200-533 ms) and long (866-1200 ms) RSIs. The range of RSI values for short and long RSI trials reflects the upper and lower tertiles of all possible RSI values. Binning RSIs into tertiles and analyzing the upper and lower tertiles allowed us to maximize signal-to-noise ratio as well as increase the likelihood of identifying an effect of RSI, given that these time ranges are consistent with previous work by Jentzsch and Dudschig (2009). A pair of repeated-measures $t$ tests were used to test whether PES and PEA differed at short relative to long RSIs. For display purposes only, PES and PEA were also calculated and plotted for the middle RSI bin (534-865 ms); however, no statistics were calculated for the middle RSI bin, given the a priori results of Jentzsch and Dudschig (2009).

\section{EEG processing}

Following data acquisition, all in-cap electrodes were rereferenced to the average of the left and right mastoid recordings and linearly detrended to remove large drifts. Data were low-pass filtered at $40 \mathrm{~Hz}$, using a Butterworth filter (Lopez-Calderon and Luck, 2010) and then down-sampled to $500 \mathrm{~Hz}$. To remove ocular artifacts and additional noise, independent component analysis (ICA) decomposition was run on an identical dataset to the one used for ERP analyses, with the addition of a $1 \mathrm{~Hz}$ high-pass filter (Debener et al., 2010). Before ICA decomposition, this dataset was segmented into $1000 \mathrm{~ms}$ epochs; to improve ICA decomposition, an initial automated rejection of noisy EEG data was performed using a combined voltage threshold rejection of $\pm 1000 \mu \mathrm{V}$ to remove disconnected channels and spectral threshold rejection using a $30 \mathrm{~dB}$ threshold within the $20-40 \mathrm{~Hz}$ band to remove EMG-like activity (EEGLAB pop_rejspec function) (Delorme and Makeig, 2004). If artifact rejection led to $>20 \%$ of epochs being rejected for a given channel, this channel was removed from both the $1 \mathrm{~Hz}$ high-pass dataset and the $0.1 \mathrm{~Hz}$ highpass ERP dataset; missing channels were not interpolated before ICA decomposition. Following ICA decomposition, identified ICA components in the $1 \mathrm{~Hz}$ high-pass filtered dataset were then copied to the original, $0.1 \mathrm{~Hz}$ high-pass ERP dataset; all further analyses were performed on the $0.1 \mathrm{~Hz}$ high-pass ERP dataset.

The $0.1 \mathrm{~Hz}$ high-pass filtered ERP dataset was epoched from -200 to $800 \mathrm{~ms}$ relative to all stimulus and response markers. Automated detection and removal of artifactual independent components, using combined analysis of spatial and temporal features, were accomplished using the ADJUST toolbox (Mognon et al., 2011). Following removal of artifactual independent components, automated rejection of any remaining noisy EEG data was performed using a voltage threshold rejection of $\pm 100 \mu \mathrm{V}$. If the threshold rejection led to $>10 \%$ of epochs being rejected for a given channel, this channel was removed from the dataset. Any missing channels were then interpolated using spherical interpolation. Next, spherical spline surface Laplacian (current source density; CSD) estimates were calculated using the CSD toolbox; electrode locations were defined using the standard montage defined by the CSD toolbox, "10-5-System_Mastoids_EGI129.csd" (Kayser and Tenke, 2006). All subsequent ERP analyses were performed on the surface Laplacian estimates $\left(\mu \mathrm{V} / \mathrm{m}^{2}\right)$ and not the raw voltage waveforms $(\mu \mathrm{V})$. Stimulus and response-locked ERP waveforms were baseline corrected to a -200 to 0 ms baseline period.

The Laplace transform was used to separate cortical sources and to attenuate volume conduction (Kayser and Tenke, 2006). The attenuation of volume conduction is especially important at short RSIs, given the temporal overlap in error-related processing in frontoparietal cortical regions and subsequent stimulus-related processing in lateral-occipital regions. Through the use of the Laplace transform, it is possible to separate this activity; this assumption was confirmed by testing for significant differences in the prestimulus baseline at short and long RSIs (see ERP analyses and Results).

\section{ERP analyses}

Stimulus-locked P1. Similar to traditional analyses of post-error behavior (Dutilh et al., 2012), analysis of stimulus-locked ERP data consisted only of correct trials that were preceded by either a correct or error response. The electrode location where the P1 component was maximal was identified in the grand-average ERP waveforms (collapsed across all subjects and conditions). In line with previous work, this process identified a peak over lateral-occipital cortex (electrode PO8) at $144 \mathrm{~ms}$ after stimulus. Therefore, for each condition of interest, the P1 component was quantified as mean $\mu \mathrm{V} / \mathrm{m}^{2}$ during a $40 \mathrm{~ms}$ window (124-164 ms) at electrode PO8 and PO7 (electrode PO7 was including in the analysis to explore any possible laterality effects). To test whether task-related attention was 
impaired following errors, the P1 component was analyzed using a threeway, previous accuracy (correct, error) by RSI (short, long) by electrode (PO7, PO8) ANOVA, with current correct trial P1 mean $\mu \mathrm{V} / \mathrm{m}^{2}$ (124$164 \mathrm{~ms})$ at PO7 and PO8 as the dependent variable. Consistent with the behavioral analyses, short RSIs were defined as ranging from 200 to 533 $\mathrm{ms}$, and long RSIs were defined as ranging from 866 to $1200 \mathrm{~ms}$. Similar to the behavioral analyses, only short and long RSI bins were analyzed for the $\mathrm{P} 1$ component. For display purposes only, the $\mathrm{P} 1$ was also calculated and plotted for the middle RSI bin (534-865 ms); however, no statistics were calculated for the middle RSI bin, given the a priori results of Jentzsch and Dudschig (2009).

To rule out the possibility that differences in the P1 component, as a function of previous trial accuracy and RSI, were due to baseline differences between conditions, the prestimulus baseline period $(-200$ to 0 $\mathrm{ms}$ ) was statistically compared before baseline correction. The prestimulus baseline period was analyzed using a two-way, previous accuracy (correct, error) by RSI (short, long) ANOVA, with current correct trial mean $\mu \mathrm{V} / \mathrm{m}^{2}$ baseline activity ( -200 to $0 \mathrm{~ms}$ ) collapsed across PO7 and PO8 as the dependent variable; analysis of the baseline condition was collapsed across electrode location, given that no interaction with electrode location was identified for the P1 component (see Results).

Another possible interpretation of any P1 decrement following errors could be that errors tend to occur during periods of the task in which overall attention has lapsed, or during periods of mind wandering, which could lead to a nonspecific decrement in the P1 component. Similarly, it is possible that a reduction in the $\mathrm{P} 1$ following errors could be due to a continuation of the process that caused the error in the first place, as opposed to a direct result of error detection per se. To rule out the possibility that any contextual (post-error) effects on the P1 component are due to general lapses in attention, a set of control analyses were conducted to investigate the P1 on error trials (in addition to the posterror P1 analyses described above). If a reduction in the P1 following errors is due to a general lapse in attention, then a P1 reduction should be observable not only on post-error trials (vs post-correct trials) but also on error (vs correct) trials. Additionally, if a post-error reduction in the P1 can simply be attributable to the continuation of a process that led to the error in the first place, then a comparison of the error (minus correct) P1 should yield and equal or larger (more negative) difference score than the post-error (minus post-correct) P1. These alternative hypotheses were tested by first comparing the error and correct trial P1 for short RSI trials that preceded correct trials using a paired-samples $t$ test; the P1 component was quantified as mean $\mu \mathrm{V} / \mathrm{m}^{2}$ during a $40 \mathrm{~ms}$ window (124-164 ms), averaged over electrodes PO8 and PO7. The lack of a significant difference for the $\mathrm{P} 1$ on error trials would be in line with the notion that the post-error $\mathrm{P} 1$ reduction is not driven by the process that caused the error, such as mind wandering or an attentional lapse. A more explicit test of whether the post-error P1 reduction was driven by the cognitive deficit that caused the error was conducted by first computing the difference between error and correct trial P1 (for short RSI trials that preceded correct trials) as well as the difference between post-error and post-correct trial P1 (for short RSI correct trials); these difference scores were then compared with a paired samples $t$ test. If the post-error P1 reduction were to be driven by an attentional lapse, then the $\mathrm{P} 1$ difference score should at least be equal to, or larger than (more negative), the post-error P1 reduction. In contrast, if the post-error P1 reduction revealed a larger magnitude change, then this would support the notion that error detection itself, not the process that led to the error, caused the post-error $\mathrm{P} 1$ reduction. It should be noted that the latter interpretation would also derive support from investigating the single-trial relationship between the Pe and the post-error P1 (as described below).

Response-locked ERN and Pe. Mirroring the analysis of stimuluslocked and behavioral data, analysis of response-locked data consisted of only correct or error responses that were followed by a correct response. To identify the ERN and Pe, a difference wave between response-locked error and correct trials was calculated. The ERN was identified as being maximal at electrode $\mathrm{Cz}$, with a peak at $22 \mathrm{~ms}$ after response; ERN amplitude was therefore calculated using a $40 \mathrm{~ms}$ window $(2-42 \mathrm{~ms})$ at electrode $\mathrm{Cz}$. The Pe was identified as being maximal at electrode $\mathrm{Pz}$, with a peak at $448 \mathrm{~ms}$ after response; Pe amplitude was calculated using a 100

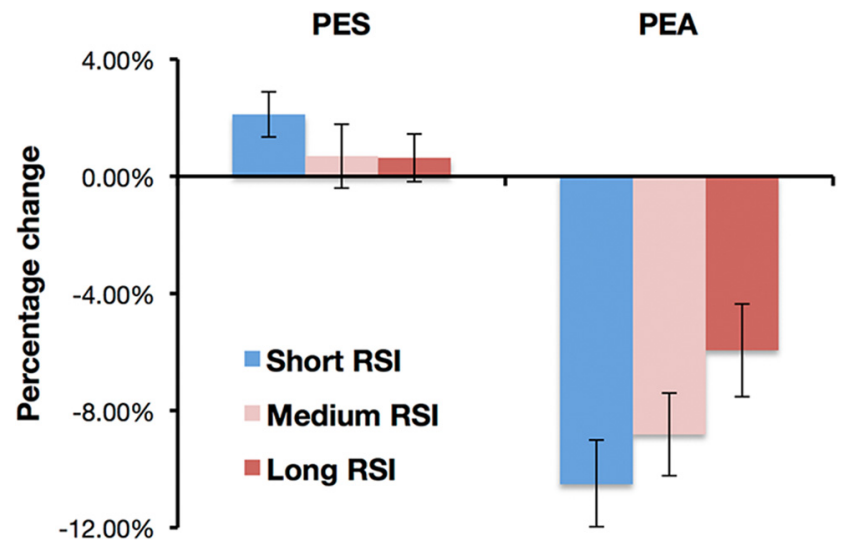

Figure 2. Post-error behavior. PES and PEA at short, medium, and long RSIs. PES and PEA were quantified as a percentage change, relative to postcorrect behavior. Error bars indicate SEM.

ms window (398-498 ms) at electrode Pz. Testing for significant ERN and Pe accuracy effects was performed to confirm that these components were predictably modulated as a function of task performance in the current study; they were investigated with a pair of repeated-measures $t$ tests comparing error and correct responses.

\section{Analyses of brain-behavior relationships}

To test whether error-related processing (Pe/ERN) was predictive of posterror attention (P1 modulation) and behavior (PES, PEA) on a single-trial level, we conducted a series of generalized linear mixed-effects analyses using the R statistical software, version 3.3.1 (R Core Team, 2016) in conjunction with the packages lme4, version 1.1-12 (Bates et al., 2015) and lmerTest, version 2.0-32 (Kuznetsova et al., 2016). As distributions of single-trial response time were observed to be positively skewed, response times were transformed to the natural logarithm of the response time to more closely approximate a normal distribution. Within each model, continuous variables were centered and scaled to have a mean of 0 and SD of 1 across the dataset, whereas categorical variables were entered using sum contrasts.

Models predicting continuous outcome measures (P1 magnitude, response time) were fit using linear mixed-effects models using the lmer function from the package lme4, with restricted maximum likelihood estimation. Models predicting binary outcome measures (accuracy) were fit using generalized linear mixed-effects models using the glmer function with logit link, from the package lme4, with maximum likelihood estimation. Each model was fit with effects of interest and their interactions (plus an intercept) as fixed effects, with subject specific variation in intercept as a random effect. Statistical significance for each fixed effect was calculated via lmerTest (Kuznetsova et al., 2016), using the Satterthwaite's approximation to denominator degrees of freedom.

The primary analysis investigated the effect of previous trial ERP component magnitude (either Pe or ERN) and RSI bin (short vs long) on next trial activity, with separate models for next trial P1 magnitude, next trial response time, and next trial accuracy. All analyses were restricted to current trial errors, whereas the analyses of next trial P1 magnitude and next trial response time were additionally restricted to pairs of trials in which the trial following the error was correct.

\section{Results}

Behavioral data

Overall accuracy was $78.16 \%(\mathrm{SE}=1.58 \%)$. Mean correct trial RT was $613 \mathrm{~ms}(\mathrm{SE}=16.64 \mathrm{~ms})$, whereas mean error RT was 607 $\mathrm{ms}(\mathrm{SE}=26.69 \mathrm{~ms})$; correct and error RTs did not differ significantly $\left(t_{(1,22)}=0.54, p=0.593, d=0.114\right)$. A trend for significantly greater PES at short RSIs $(2.12 \%, \mathrm{SE}=0.77 \%)$, compared with long RSIs $(0.63 \%, \mathrm{SE}=0.81 \%)$ was observed $\left(t_{(1,22)}=1.80\right.$, $p=0.086, d=0.77)$. Similarly, PEA was significantly lower at short RSIs $(-10.52 \%, \mathrm{SE}=1.51 \%)$, compared with long RSIs $(-5.94 \%$, 
A

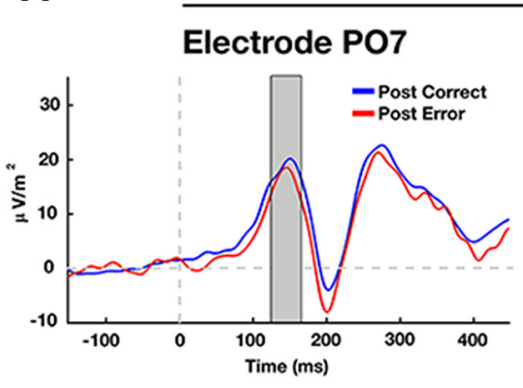

Short RSI

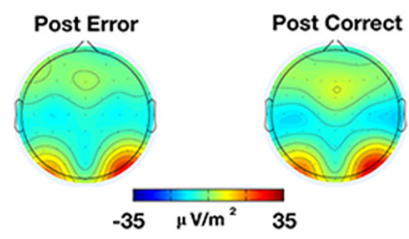

Post Error - Post Correct

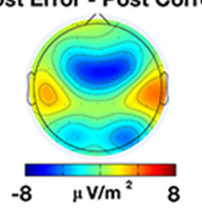

B

Medium RSI
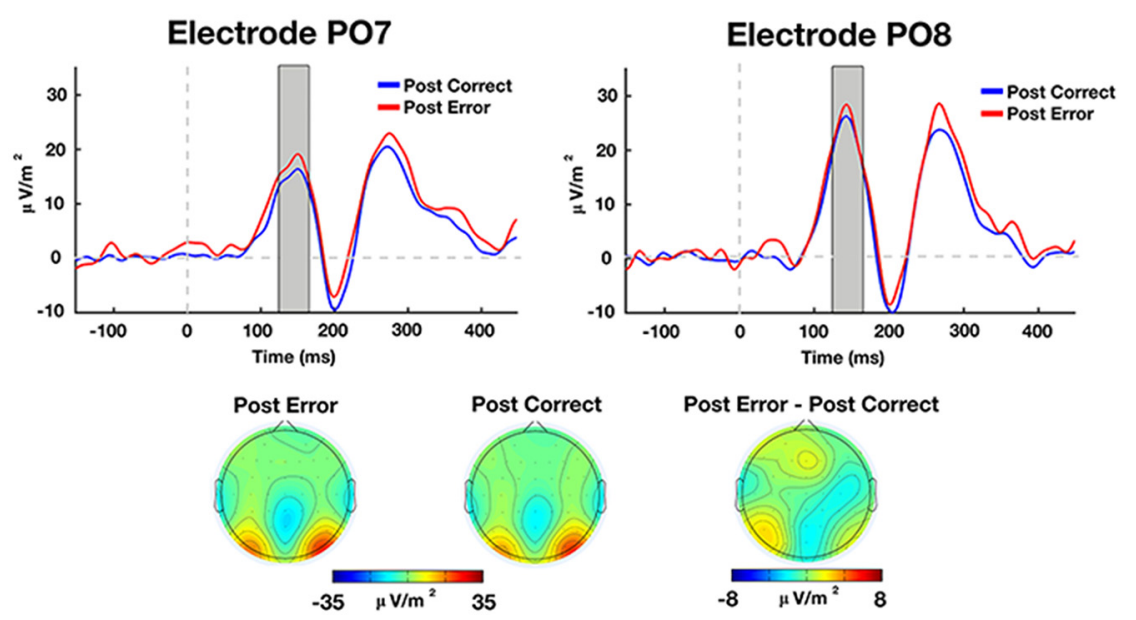

C

Long RSI
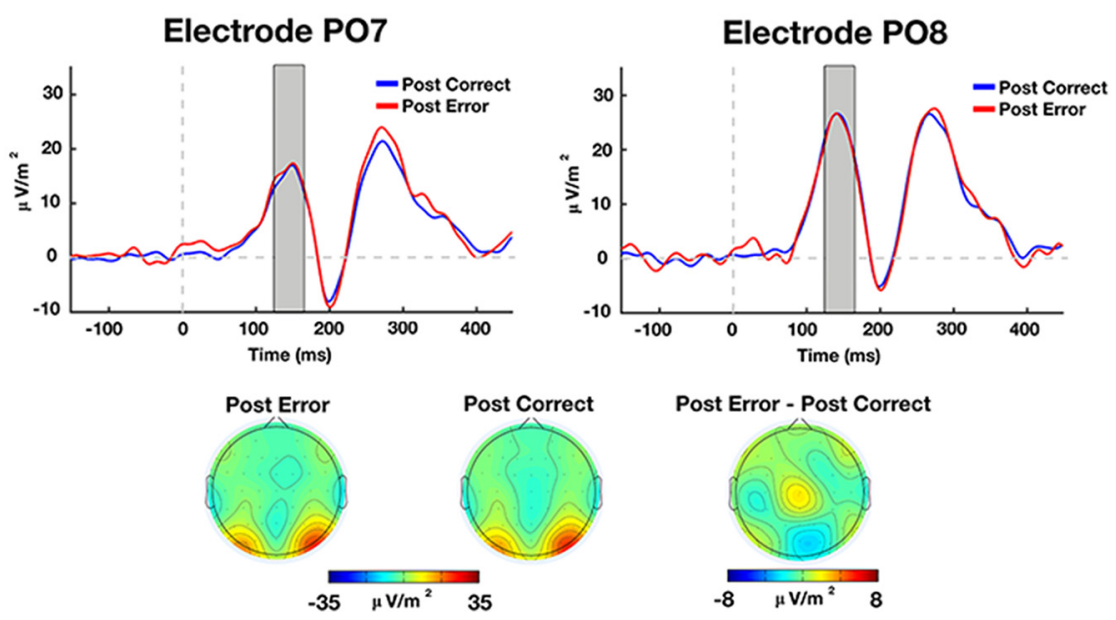

Figure 3. P1 modulation as a function of previous accuracy and RSI. P1 ERP components and topographic plots for the P1 component at electrodes P07 and P08 following $(\boldsymbol{A})$ short, $(\boldsymbol{B})$ medium, and $(\boldsymbol{C})$ long RSIs. Shaded regions represent the analysis window of the $P 1$ component. Topographic plots represent average amplitude during the analysis window.

$\mathrm{SE}=1.58 \%)\left(t_{(1,22)}=2.51, p=0.02, d=1.07\right)$. This pattern of results is in line with the notion that errors can lead to distraction, especially when RSIs are short (for a depiction of the behavioral results, see Fig. 2).

\section{Electrophysiology}

Stimulus-locked P1

Analysis of the $\mathrm{P} 1$ component revealed a main effect of RSI $\left(F_{(1,22)}=7.01, p=\right.$ $\left.0.015, \chi_{p}^{2}=0.242\right)$. Additionally, a main effect of electrode was identified, with the P1 component being larger at electrode PO8 $\left(F_{(1,22)}=9.02, p=0.007, \chi_{p}^{2}=\right.$ 0.291 ); electrode location did not significantly interact within any other effects. Critically, an interaction between previous accuracy and RSI was near significant $\left(F_{(1,22)}=4.22, p=0.052, \chi_{p}^{2}=0.161\right)$. This interaction demonstrates that, beyond any main effect of RSI, errors led to greater reduction of the $\mathrm{P} 1$ at short, but not long RSIs. Given that the $p$ value for the interaction between accuracy and RSI was only near significant, follow-up $t$ tests were corrected for multiple comparisons using a Bonferroni corrected alpha value of 0.025. Follow-up $t$ tests (collapsed across PO7 and PO8) revealed that the P1 component was significantly smaller following errors $(21.68, \mathrm{SE}=3.02)$, compared with correct responses $(24.33, \mathrm{SE}=3.23)$, at short RSIs $\left(t_{(1,22)}=2.45, p=0.023, d=1.04\right)$. In contrast, the P1 component was not significantly different following error (19.89, SE = 3.19) and correct (19.68, $\mathrm{SE}=3.26)$ responses at long RSIs $\left(t_{(1,22)}\right.$ $=0.21, p=0.837, d=0.09)$. This pattern of results is consistent with the notion that errors cause an attentional bottleneck, which leads to impaired sensory processing at short RSIs (for a depiction of the P1 effects, see Fig. 3).

Critically, there was no significant interaction between previous trial accuracy and RSI during the prestimulus (posterror) baseline period at electrodes $\mathrm{PO} 7$ and $\operatorname{PO} 8\left(F_{(1,22)}=0.19, p=0.668\right)$. This null result suggests that the $\mathrm{P} 1$ effect cannot be due to differences in the prestimulus baseline. Further, these results confirm that the use of a Laplace transform was successful in isolating stimulus-related neural activity at lateral-occipital electrode sites from ongoing error-related activity at frontoparietal electrode locations.

Comparison of the error and correct trial P1 (at short RSIs, before correct trials) demonstrated no significant difference $\left(t_{(1,22)}=0.17, p=0.865, d=0.07\right.$; Fig. 4). Additionally, it was found that the post-error minus post-correct $\mathrm{P} 1$ difference score $(-2.65, \mathrm{SE}=1.08)$ was significantly more negative than the error minus correct P1 difference score for short RSI trials that preceded correct trials $(0.12, \mathrm{SE}=0.73)\left(t_{(1,22)}=2.25, p=0.035, d=0.95\right)$. These control analyses provide crucial evidence that the post-error P1 reduction is not the result of either a general lapse in attention or 
mind wandering. If the post-error $\mathrm{P} 1$ were the result of a general lapse in attention, then this would be observable as a reduction in the $\mathrm{P} 1$ on both post-error and error trials. Further, if the post-error reduction in the $\mathrm{P} 1$ were the result of a continuation of the cognitive deficit that caused the error, then the error trial difference score would be equal to, or more negative than, the post-error P1. In direct contrast, the error trial P1 difference score was more positive than the post-error trial P1 difference score; this pattern of results is consistent with the notion that errors lead to an error detection process, which in turn, causes impaired sensory processing on the subsequent trial.

Response-locked ERN and Pe

The presence of a significant accuracy effect for the ERN and Pe was tested with a pair of repeated-measures $t$ tests comparing error and correct responses (regardless of whether the subsequent RSI was long or short) (for a depiction of the ERN and Pe, see Fig. 5). The ERN was significantly more negative on error $(-9.24, \mathrm{SE}=1.87)$, compared with correct $(-4.82, \mathrm{SE}=1.78)$ responses $\left(t_{(1,22)}=2.88, p=0.009, d=1.23\right)$. The Pe was significantly more positive on error $(0.89, \mathrm{SE}=2.38)$, compared with correct $(-6.81, \mathrm{SE}=2.43)$ responses $\left(t_{(1,22)}=4.71, p<0.001, d=\right.$ 2.01).

\section{Relationship between error-monitoring and post-error trial $\mathrm{Pe}$}

We tested whether either of the error-related components (ERN and $\mathrm{Pe}$ ) was predictive of task performance following errors ( $\mathrm{P} 1$, PEA, PES), at short and long RSIs. Critically, the linear mixedeffects model of Pe and RSI on next trial P1 revealed a significant interaction between $\mathrm{Pe}$ and RSI bin (estimate $=0.064$, SE $=$ $0.032, \mathrm{df}=3392.27, t=2.03, p=0.042$ ). Specifically, the Pe was associated with a reduced $\mathrm{P} 1$ component on post-error trials, but the negative relationship between the Pe and $\mathrm{P} 1$ was only present at short RSI bins (Fig. 6). No other significant main effects or interactions were identified for this model (all $p>0.10$ ). Together with literature linking the Pe to conscious deliberation over whether an error has occurred (Nieuwenhuis et al., 2001; Steinhauser and Yeung, 2010), the Pe-P1 correlation suggests that the depth of error processing directly predicts whether errors will be distracting at short RSIs.

The linear mixed-effects model of Pe and RSI on the logarithm of next trial RT revealed a significant main effect of Pe (estimate $=-0.055, \mathrm{SE}=0.016, \mathrm{df}=3403.10, t=-3.41, p=0.001$ ) such that a larger Pe was associated with a faster response on the next trial, and a significant main effect of RSI bin (estimate $=$ $-0.170, \mathrm{SE}=0.030, \mathrm{df}=3390.21, t=-5.61, p<0.001)$ such that the longer RSI period was associated with a faster response on the next trial, with no interaction between Pe and RSI $(p>0.6)$.

The linear mixed-effects model of Pe and RSI on the likelihood of next trial accuracy revealed a significant main effect of RSI (estimate $=0.264, \mathrm{SE}=0.064, z=4.11, p<0.001$ ) such that the longer RSI period was associated with a higher likelihood of responding to the next trial correctly, whereas neither the main

\section{Current Trial P1 - Control Analysis}

Electrode PO7

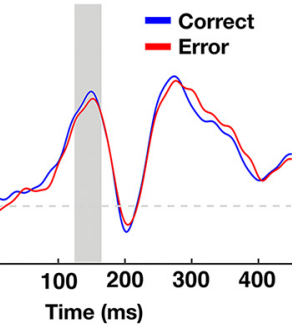

Error

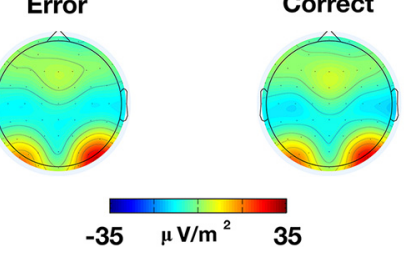

\section{Electrode PO8}

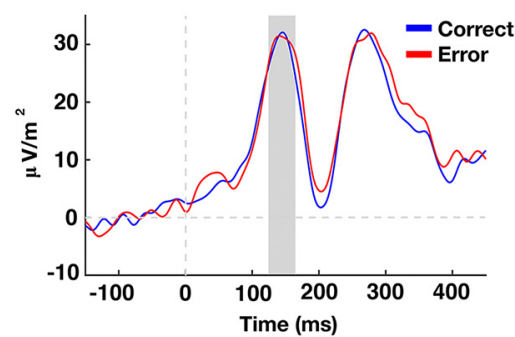

Error - Correct

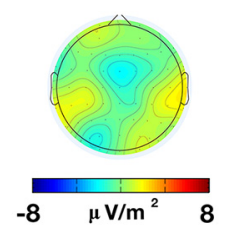

effect of Pe nor the interaction between Pe and RSI was significant (both $p>0.4$ ).

\section{ERN}

The linear mixed-effects model of ERN and RSI on next trial P1 revealed a significant main effect of ERN magnitude on next trial $\mathrm{P} 1$ magnitude (estimate $=0.034, \mathrm{SE}=0.016, \mathrm{df}=3403.23, t=$ 2.10, $p=0.036$ ) such that a smaller (more positive) ERN was associated with a more positive P1 on the next trial. Neither the main effect of RSI nor the ERN by RSI interactions was significant (both $p>0.1$ ).

The linear mixed-effects model of ERN and RSI on the logarithm of next trial RT revealed a significant main effect of RSI bin (estimate $=-0.171, \mathrm{SE}=0.030, \mathrm{df}=3390.22, t=-5.64, p<$ 0.001 ) such that the longer RSI period was associated with a faster response on the next trial, with no main effect of ERN magnitude and no interaction between ERN and RSI (both $p>0.8$ ).

The linear mixed-effects model of ERN and RSI on the likelihood of next trial accuracy revealed a significant main effect of RSI (estimate $=0.268, \mathrm{SE}=0.064, z=4.16, p<0.001$ ) such that the longer RSI period was associated with a higher likelihood of responding to the next trial correctly, whereas neither the main effect of ERN nor the interaction between ERN and ITI was significant (both $p>0.06$ ).

\section{Control analyses of the $\mathrm{Pe}-\mathrm{P} 1$ relationship}

It is unlikely that the Pe-P1 relationship reflects an artifact of shared volume conduction. First, a CSD transform was explicitly used to separate the activity of these two components. Second, given that the $\mathrm{Pe}$ and $\mathrm{P} 1$ are both positive deflections, if shared volume conduction contributed to their relationship, then this would be expected to manifest as a positive correlation, inconsistent with the observed negative correlation. Nonetheless, to provide further evidence against the possibility of shared volume conduction driving the $\mathrm{Pe}-\mathrm{P} 1$ relationship, we conducted a linear mixed-effects model of $\mathrm{Pe}$ and RSI on the next trial (post-error) baseline period. Critically, the mixed-effects model revealed no interaction between Pe and RSI in terms of predicting the next trial (post-error) baseline 

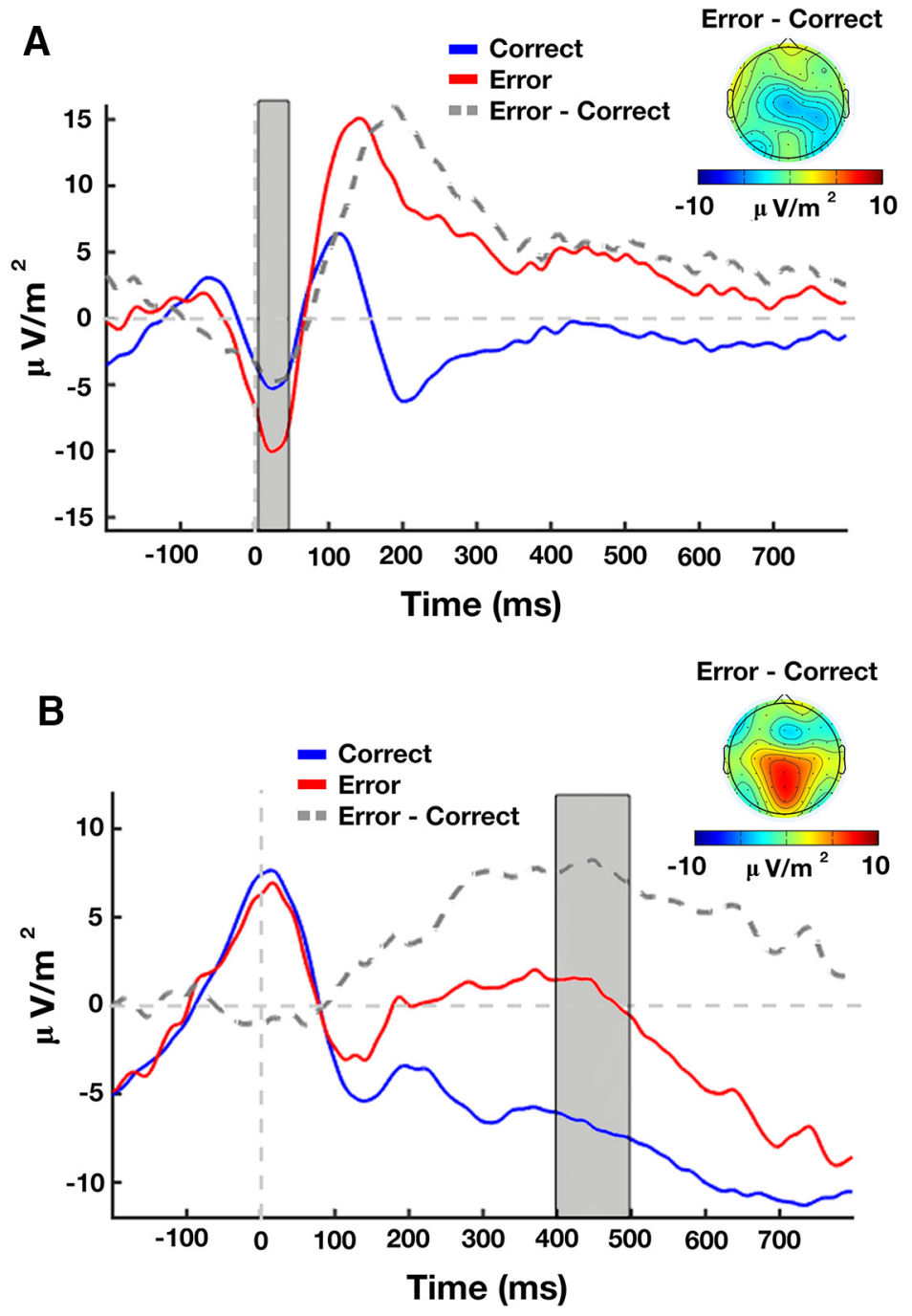

Figure 5. Response-locked error-related ERP activity. ERP and topographic plots of the ( $\boldsymbol{A})$ ERN and $(\boldsymbol{B})$ Pe. Shaded regions represent the analysis window of the ERP components. Topographic plots represent average amplitude of the difference wave during the analysis window.

period $(p>0.3)$. This result provides further evidence that the Pe-P1 relationship is not due to a nonspecific contribution of shared volume conduction.

We also considered the possibility that the Pe-P1 relationship could be explained by periods of mind wandering, leading to erroneous responses, which then carry over to the subsequent (post-error) trial. To rule out this possibility, we conducted a mixed-effects model in which the stimulus-locked P1 component occurring on error trials and RSIs were used to predict the posterror P1 component. Measurement of the stimulus-locked P1 component on the error trial allowed for a measure of taskrelated attention before error commission. The P1 component on the error trial was quantified as mean amplitude during a 40 ms window (124-164 ms), collapsed across electrodes PO7 and PO8. Critically, no interaction between the P1 on error trials and RSI was identified ( $p>0.9)$. This result provides strong evidence that the Pe-P1 relationship is indicative of error detection causing an attentional bottleneck on the subsequent trial and that the Pe-P1 correlation cannot be explained by an overall lapse in attention leading to both error commission and subsequent attentional impairment.

\section{Discussion}

The present study provides evidence that detection of errors can lead to impairment of task-related sensory processing on subsequent trials. At short RSIs, a marked reduction in both post-error accuracy and task-related sensory processing, as indexed by P1, was observed. Moreover, single-trial variation in the Pe component, a well-established index of error processing, interacted with RSI length to predict P1 diminution on post-error trials. Critically, control analyses of the P1 on error trials demonstrated that this effect could not be due to an overall lapse in attention or mind wandering. Rather, these results suggest that the error detection process itself leads to an attentional bottleneck, due to resources being devoted to deciding whether an error was made (Steinhauser and Yeung, 2010). In turn, this attentional bottleneck appears to cause active suppression of task-relevant sensory processing on the subsequent trial, as indexed by a reduced $\mathrm{P} 1$ component. The present results provide strong evidence that errors do not categorically lead to increased control and are suggestive of competing processes of distraction and control following errors.

The observed pattern of behavioral results, as a function of RSI, is consistent with recent behavioral studies investigating the influence of RSI on post-error behavior. Specifically, when RSIs are $<\sim 500 \mathrm{~ms}$, increased PES and reduced PEA have been observed (Jentzsch and Dudschig, 2009; Houtman and Notebaert, 2013; Van der Borght et al., 2016a). The current results extend previous behavioral and electrophysiological work in several important ways. First, the present study manipulated RSI within blocks, preventing a change in global strategy or confound of arousal across blocks. Second, although recent work by Van der Borght et al. (2016b) demonstrated that sensory attention can become impaired following errors, we note that they assessed sensory attention in a task orthogonal to the one in which errors occurred. Our study used a single-task paradigm, allowing for within-task comparisons of error processing and sensory attention. Third, in contrast to Van der Borght et al. (2016b), we found that error-related reductions in sensory processing were specific to short RSIs. Finally, the current study demonstrated that single-trial measures of error processing are predictive of subsequent impairments in sensory attention.

The single-trial relationship between the error-evoked Pe and the subsequent stimulus-locked P1 suggests a mechanism driving distraction after errors. The Pe has been argued to reflect a resourceintensive process associated with error detection and awareness (Nieuwenhuis et al., 2001). Specifically, there is evidence that the Pe reflects either the motivational salience of an error (Ridderinkhof et al., 2009) or accrual of evidence to decide whether an error was made 


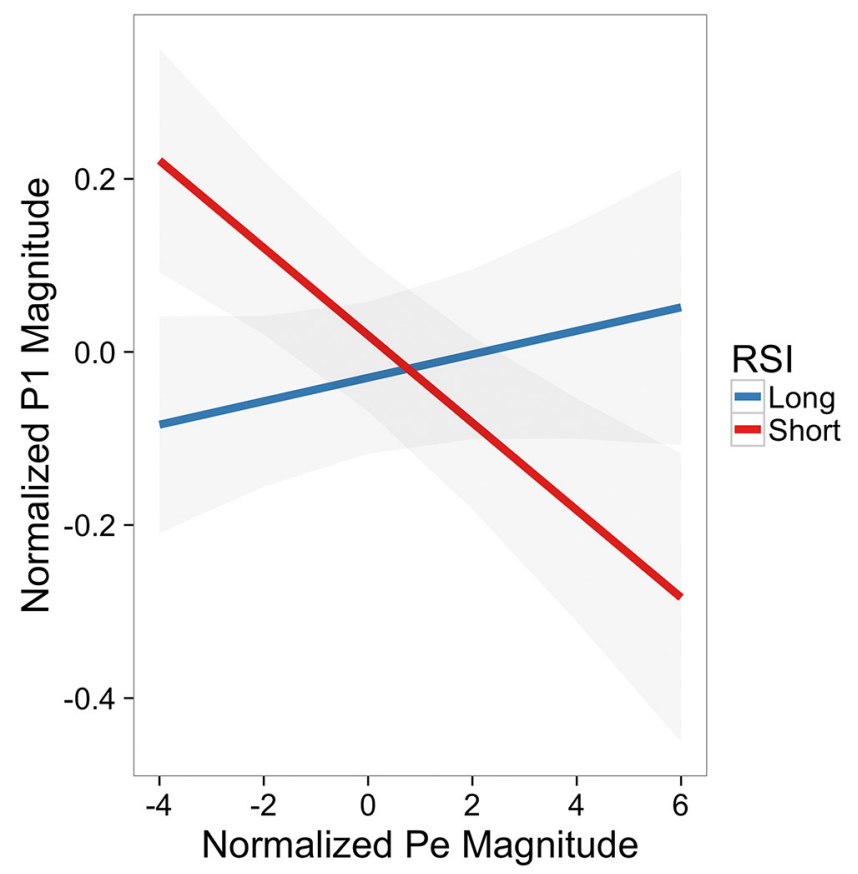

Figure 6. Relations between single-trial Pe, P1, and RSI. Predicted values for the post-error $P 1$, relative to error-evoked Pe and RSI bin (short vs long), derived from the linear mixed-effects model. Error-evoked Pe and RSI bin interact, such that the influence of error-evoked Pe magnitude on subsequent trial P1 differs between short and long RSI bins. Increased Pe magnitude only leads to a reduced P1 magnitude when the RSI is short. Shaded region around each line represents \pm SE. Values were derived using the "effects" package for $R$ (Fox, 2003).

(Steinhauser and Yeung, 2010); both of these processes likely place a high demand on central resources. We therefore suggest that distraction at short RSIs is the direct result of central resources being taxed by error-related processing, consistent with work demonstrating that selective attention can be impaired when central resources are occupied (Lavie, 1995; de Fockert et al., 2001). In line with this notion, the general time course during which the Pe component was maximal (398-498 ms after response) is consistent with the time range in which distraction occurred (200-533 ms after response).

One possible explanation for a reduced P1 following errors may be that deciding whether an error was made limits the ability to implement top-down control over visual cortex on subsequent trials; that is, P1 reduction may reflect a failure to enhance sensory processing. Alternatively, the motivational salience of errors (Ridderinkhof et al., 2009) may lead to prioritization of error-related processing, through active suppression of other task-related processes, including stimulus processing (P1) on the next trial. Within this account, $\mathrm{P} 1$ reduction may reflect active suppression of sensory processing. Although the current results are not able to provide conclusive evidence for either of these possibilities, recent ERP investigations of attention increasingly link P1 modulation to an active process of attentional suppression (Moher et al., 2014; Slagter et al., 2016). Additionally, control analyses in the current study demonstrate that error commission did not arise due to modulation of early sensory processing, indexed by the $\mathrm{P} 1$ component, but instead must arise at a later stage of processing, consistent with other ERP investigations of perceptual discrimination of foveally presented stimuli (Handy and Khoe, 2005; Fedota et al., 2012). Together, these data suggest that error processing causes an attentional bottleneck, which then leads to prioritization of error-related information through the active suppression of task-relevant information on the subsequent trial. However, future research will be needed to provide evidence confirming this hypothesis.
The primary finding of the current study is that trial-by-trial fluctuations in the magnitude of error-related processing, as indexed by the Pe, are negatively related to sensory processing on the subsequent trial, but only when RSIs are short. This relationship between error processing and subsequent sensory processing was demonstrated by a significant interaction between single-trial Pe magnitudes and RSI length, when predicting subsequent trial P1 amplitude. It should be noted that the relationship between singletrial Pe amplitudes and behavior on the subsequent trial was also investigated; similarly, the relationship between single-trial ERN amplitudes and next trial behavior were analyzed. However, none of these additional analyses revealed a significant interaction with RSI length, suggesting that all other results are orthogonal to the primary finding that error detection (as measured by the Pe) leads to impaired sensory processing when RSIs are short. Nonetheless, one effect worth discussing is the negative relationship between singletrial Pe amplitude and PES on the subsequent trial. Whereas PES has traditionally been interpreted as a behavioral signature of the application of top-down control (Botvinick et al., 2001), more recent research has suggested that the utility of PES may vary as a function of RSI (Jentzsch and Dudschig, 2009). Specifically, behavioral evidence from humans (Jentzsch and Dudschig, 2009) and both behavioral and physiological evidence from primates (Purcell and Kiani, 2016) suggest that PES may reflect distraction at short RSIs, likely due to attentional orienting (Notebaert et al., 2009), and control at longer RSIs. At the group level, we found that PEA was lowest at short RSIs and paired with a nonsignificant trend for PES to be largest at short RSIs. This pattern of behavioral results at the group level is generally in line with prior research and the notion that the functional significance of PES may vary with RSI length. However, the single-trial analysis of Pe amplitude yielded an overall negative relationship with PES, an effect that did not interact with RSI. Critically, the fact that the Pe-P1 relationship, but not the relationship between Pe and PES, interacted with RSI suggests that at least two separable processes follow error commission (Danielmeier and Ullsperger, 2011; Maier et al., 2011). The current study elucidates one process that follows error commission: a short-lasting impairment in sensory processing, due to attentional bottleneck caused by error processing. However, an increased understanding into the dynamics of PES will require additional research. Indeed, a recent model of post-error behavior suggests that PES may vary across tasks (Danielmeier and Ullsperger, 2011; Ullsperger and Danielmeier, 2016). Given that the current study used a sensory discrimination task, as opposed to the more common investigations of PES that use response-conflict tasks, additional research in which both perceptual and response conflict tasks are assessed in a within-subjects design will be required to build a more general model of the cognitive and behavioral processes that follow error commission.

Previous work using tasks with long RSIs has provided evidence that errors can lead to enhanced activation in task-relevant cortical regions (King et al., 2010; Danielmeier et al., 2011) and increased accuracy (Maier et al., 2011) when RSIs are $>\sim 1000$ ms. In the present study, no evidence for increased control was observed in terms of behavior (PES, PEA) or task-related attention (P1 modulation), even at long RSIs. One explanation for the discrepancy across studies is that control following errors is more predominant within tasks that involve a high degree of response conflict, such as the Eriksen flanker task (Eriksen and Eriksen, 1974). Alternatively, it is possible that, at least for perceptual discrimination tasks, instantiation of control following errors requires even longer RSIs.

In conclusion, the present results demonstrate that error detection can directly lead to impairment in task-related attention 
when RSIs are relatively short ( $<533 \mathrm{~ms}$ ). Critically, the depth of error processing, as indexed by the $\mathrm{Pe}$, predicts the degree to which task-related attention (P1) is impaired on subsequent trials. These results suggest that error processing is a resourceintensive process, which creates an attentional bottleneck; that is, the very system dedicated to monitoring and improving ongoing behavior can sometimes be the source of impaired performance.

\section{References}

Bates D, Maechler M, Bolker B, Walker S (2015) Fitting linear mixed-effects models using lme4. J Stat Software 67:1-48.

Botvinick MM, Braver TS, Barch DM, Carter CS, Cohen JD (2001) Conflict monitoring and cognitive control. Psychol Rev 108:624-652. CrossRef Medline

Brainard DH (1997) The psychophysics toolbox. Spat Vis 10:433-436. CrossRef Medline

Danielmeier C, Ullsperger M (2011) Post-error adjustments. Front Psychol 2:233. CrossRef Medline

Danielmeier C, Eichele T, Forstmann BU, Tittgemeyer M, Ullsperger M (2011) Posterior medial frontal cortex activity predicts post-error adaptations in task-related visual and motor areas. J Neurosci 31:1780-1789. CrossRef Medline

Debener S, Thorne J, Schneider TR, Campos Viola F (2010) Using ICA for the analysis of multi-channel EEG Data. In: Simultaneous EEG and fMRI: recording, analysis, and application, pp 121-133. Oxford: Oxford UP.

de Fockert JW, Rees G, Frith CD, Lavie N (2001) The role of working memory in visual selective attention. Science 291:1803-1806. CrossRef Medline

Delorme A, Makeig S (2004) EEGLAB: an open source toolbox for analysis of single-trial EEG dynamics including independent component analysis. J Neurosci Methods 134:9-21. CrossRef Medline

Dutilh G, Vandekerckhove J, Forstmann BU, Keuleers E, Brysbaert M, Wagenmakers EJ (2012) Testing theories of post-error slowing. Atten Percept Psychophys 74:454-465. CrossRef Medline

Eriksen BA, Eriksen CW (1974) Effects of noise letters upon the identification of a target letter in a nonsearch task. Percept Psychophys 16:143-149. CrossRef

Fedota JR, McDonald CG, Roberts DM, Parasuraman R (2012) Contextual task difficulty modulates stimulus discrimination: electrophysiological evidence for interaction between sensory and executive processes. Psychophysiology 49:1384-1393. CrossRef Medline

Fox J (2003) Effect displays in R for generalised linear models. J Stat Software $8: 1-27$.

Handy TC, Khoe W (2005) Attention and sensory gain control: a peripheral visual process? J Cogn Neurosci 17:1936-1949. CrossRef Medline

Hillyard SA, Vogel EK, Luck SJ (1998) Sensory gain control (amplification) as a mechanism of selective attention: electrophysiological and neuroimaging evidence. Philos Trans R Soc Lond B Biol Sci 353:1257-1270. CrossRef Medline

Holroyd CB, Coles MG (2002) The neural basis of human error processing: reinforcement learning, dopamine, and the error-related negativity. Psychol Rev 109:679-709. CrossRef Medline

Houtman F, Notebaert W (2013) Blinded by an error. Cognition 128:228236. CrossRef Medline

Jentzsch I, Dudschig C (2009) Why do we slow down after an error? Mechanisms underlying the effects of posterror slowing. Q J Exp Psychol 62: 209-218. CrossRef Medline

Kayser J, Tenke CE (2006) Principal components analysis of Laplacian waveforms as a generic method for identifying ERP generator patterns: I.
Evaluation with auditory oddball tasks. Clin Neurophysiol 117:348-368. CrossRef Medline

King JA, Korb FM, von Cramon DY, Ullsperger M (2010) Post-error behavioral adjustments are facilitated by activation and suppression of taskrelevant and task-irrelevant information processing. J Neurosci 30: 12759-12769. CrossRef Medline

Kleiner M, Brainard D, Pelli D (2007) What's new in Psychtoolbox-3? Perception 36 ECVP Abstr Suppl.

Kuznetsova A, Brockhoff PB, Christensen RHB (2016) Package "ImerTest." R Package Version, 2. Retrieved from http://cran.uib.no/web/packages/ lmerTest/lmerTest.pdf.

Lavie N (1995) Perceptual load as a necessary condition for selective attention. J Exp Psychol Hum Percept Perform 21:451-468. CrossRef Medline

Lopez-Calderon J, Luck S (2010) ERPLAB. Davis, CA: University of California-Davis Center for Mind and Brain.

Maier ME, Yeung N, Steinhauser M (2011) Error-related brain activity and adjustments of selective attention following errors. Neuroimage 56:2339 2347. CrossRef Medline

Mognon A, Jovicich J, Bruzzone L, Buiatti M (2011) ADJUST: an automatic EEG artifact detector based on the joint use of spatial and temporal features. Psychophysiology 48:229-240. CrossRef Medline

Moher J, Lakshmanan BM, Egeth HE, Ewen JB (2014) Inhibition drives early feature-based attention. Psychol Sci 25:315-324. CrossRef Medline

Nieuwenhuis S, Ridderinkhof KR, Blom J, Band GP, Kok A (2001) Errorrelated brain potentials are differentially related to awareness of response errors: evidence from an antisaccade task. Psychophysiology 38:752-760. CrossRef Medline

Notebaert W, Houtman F, Opstal FV, Gevers W, Fias W, Verguts T (2009) Post-error slowing: an orienting account. Cognition 111:275-279. CrossRef Medline

PelliDG (1997) TheVideoToolbox software for visual psychophysics: transforming numbers into movies. Spat Vis 10:437-442. CrossRef Medline

Purcell BA, Kiani R (2016) Neural mechanisms of post-error adjustments of decision policy in parietal cortex. Neuron 89:658-671. CrossRef Medline

Ridderinkhof KR, Ullsperger M, Crone EA, Nieuwenhuis S (2004) The role of the medial frontal cortex in cognitive control. Science 306:443-447. CrossRef Medline

Ridderinkhof KR, Ramautar JR, Wijnen JG (2009) To PE or not to PE: a P3-like ERP component reflecting the processing of response errors. Psychophysiology 46:531-538. CrossRef Medline

Slagter HA, Prinssen S, Reteig LC, Mazaheri A (2016) Facilitation and inhibition in attention: functional dissociation of pre-stimulus alpha activity, P1, and N1 components. Neuroimage 125:25-35. CrossRef Medline

Steinhauser M, Yeung N (2010) Decision processes in human performance monitoring. J Neurosci 30:15643-15653. CrossRef Medline

Ullsperger M, Danielmeier C (2016) Reducing speed and sight: how adaptive is post-error slowing? Neuron 89:430-432. CrossRef Medline

Ullsperger M, Danielmeier C, Jocham G (2014) Neurophysiology of performance monitoring and adaptive behavior. Physiol Rev 94:35-79. CrossRef Medline

Van der Borght L, Braem S, Stevens M, Notebaert W (2016a) Keep calm and be patient: the influence of anxiety and time on post-error adaptations. Acta Psychol (Amst) 164:34-38. CrossRef Medline

Van der Borght L, Schevernels H, Burle B, Notebaert W (2016b) Errors disrupt subsequent early attentional processes. PLoS One 11:e0151843. CrossRef Medline

Zhang W, Luck SJ (2009) Feature-based attention modulates feedforward visual processing. Nat Neurosci 12:24-25. CrossRef Medline 